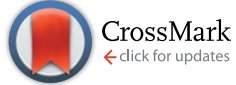

Cite this: RSC Adv., 2017, 7, 16210

Received 12th February 2017

Accepted 6th March 2017

DOI: 10.1039/c7ra01751k

rsc.li/rsc-advances

\title{
Robust ambient pressure dried polyimide aerogels and their graphene oxide directed growth of 1D- 2D nanohybrid aerogels using water as the only solvent
}

\begin{abstract}
Guangjie Yang, Tianli Ning, Wei Zhao, Wenxiu Deng and Xikui Liu*
Here, using water as the only solvent, we report the first green approach to the morphological controlled synthesis of robust ambient dried polyimide aerogels composed of interconnected hollow spheres. The addition of graphene oxide can direct the growth of polyimide into nanofibers and form 1D-2D nanohybrid aerogels. The mechanism behind is a soft/hard template directed dissolution-polymerizationreprecipitation process. After carbonization, a monolith carbon aerogel with monodisperse micropores could be formed. Such a green approach for the morphology controlled synthesis of robust polyimide aerogels and their carbon aerogels should be of great value for their environmental and energy related applications.
\end{abstract}

\section{Introduction}

Aerogels, including silica, metal oxide, chalcogenide and polymeric aerogels have attracted great scientific and industrial interest due to their three-dimensional hierarchical morphology, low densities and low thermal conductivity, and thus have great potential applications in catalysis, sensors, detectors, thermal insulators and optoelectronic applications. ${ }^{1-7}$ Meanwhile, graphene-based materials, owing to their unique structural, mechanical and electronic properties, have great potential applications in batteries, hybrid photovoltaics, biology and hydrogen storage $;^{8-12}$ graphenebased aerogels have also become a hot field. For example, Gong prepared hybrid organic aerogels consisting of polyvinyl alcohol, cellulose nanofibrils and graphene oxide nanosheets using a freeze-drying process. ${ }^{13}$ Liu also reported a polyimide-graphene-montmorillonite composite aerogel with enhanced flame-retardant performance via a freezedrying method followed by a thermal imidization process. ${ }^{14}$

The production of polymeric aerogels generally involves the direct gelation of a molecular precursor in a sol-gel process, ${ }^{15}$ extended hydrolysis and condensation beyond particle formation eventually lead to an interconnecting porous network containing a continuous liquid phase. To keep the scaffold from collapsing during the removal of the liquid phase, a supercritical drying process or freeze-drying is needed. During the gelation process and solvent exchange process, large amount of solvents that may serious damage our environment were used.

College of Polymer Science and Engineering, Sichuan University, Chengdu, 610065, P. R. China. E-mail: xkliu@scu.edu.cn
Taking account of the increasing atmospheric pollution and growing concerns on environmental issues, green process for the preparation of high performance polymeric aerogel without using any organic solvent but only water become an emergency task.

Polyimide (PI) is a kind of high performance polymer with excellent mechanical properties and thermal stability. Many researchers especially NASA have carried innovative researches on the preparation of PI aerogels using various dianhydride and multifunctional amines, ${ }^{16-18}$ and generally, high boiling point solvents like $N, N$-dimethylformamide (DMF), $N, N$-dimethylacetamide (DMAc) and $N$-methyl-2-pyrrolidone (NMP) are used to prepare the polyamic acid (PAA) precursor, followed by supercritical $\mathrm{CO}_{2}$ dying to afford PI aerogels. Most of these high boiling points are very difficult to recycle and thus cause serious environmental pollution.

Water is an environmentally friendly and benign solvent, in recent years, Unterlass, Weber and Antonietti have carried innovative studied on the synthesis of linear aromatic PIs under hydrothermal (HT) conditions, ${ }^{19-22}$ in most of the case, crystalline PI nanoplates or their flower-like aggregates were formed, and the morphological evolution over time suggests that PI crystallites grow via a dissolution-polymerization-crystallization process. Given the huge academic and industrial interest for various properties such as high modulus, high temperature stability, gas permeability, dielectric and optical properties, such a new and green synthesis routine towards high performance polyimide would be of high interest. So far, many progress are still needed, for example, most of the research were focused on linear PIs, those with highly cross-linked framework structure thus possess more excellent thermomechanical 
properties have been seldom reported. More importantly, for their industrial use, monolith morphology like aerogel is of vital importance, however, so far, almost all of the hydrothermal synthesized PIs were small powders.

Here through a soft/hard template directed hydrothermal polymerization process using nylon-salt-type monomers as precursor, we reported the first green approach to the morphological controlled synthesis of monolithic fully aromatic polyimide aerogels and their graphene nanohybrid aerogel using nothing but water. The polyimide aerogel are mechanical robust which can be directly dried in oven without significant contraction. SEM revealed the aerogel was composed with tightly interconnected hollow microspheres. With the addition of graphene oxide as hard template, an unusual polyimide nanowires were grown on the surface of GO and thus form the 1D-2D nanohybrid aerogels. A generalized mechanism based on dissolution-polymerizationreprecipitation was proposed for such a morphological controlled growth of PI aerogels. To the best of our knowledge, this is the first example of highly cross-linked, fully aromatic polyimide aerogel showing a monolithic character and high compressive strength with excellent reversibility. ${ }^{23}$

\section{Experimental}

\subsection{Materials}

Pyromellitic dianhydride (PMDA), 3,3',4,4'-biphenyltetracarboxylic dianhydride (BPDA) and 4,4'-oxydiphthalic anhydride (ODPA) were purchased from Aladdin Chemical regent Co., and vacuum dried at $200{ }^{\circ} \mathrm{C}$ for $5 \mathrm{~h}$ before use. 1,3,5-Tris(4aminophenyl) benzene (TAPB) was purchased from Zhengzhou Alfachem Co., Ltd and use as received. Graphene oxide aqueous solutions (concentration: $2 \mathrm{mg} \mathrm{ml}^{-1}, 4 \mathrm{mg} \mathrm{ml}^{-1}, 8 \mathrm{mg} \mathrm{ml}^{-1}$ ) were purchased from Carmery Materials Technology Co., Ltd. All the other solvents like ethanol, tetrahydrofuran and acetone were purchased from Chengdu Kelong chemical regent Co.

\subsection{Preparation of nylon-salt-type monomers}

In a typical experiment, $0.1709 \mathrm{~g}$ ODPA was added to $5 \mathrm{ml}$ deionized water with a magnetic stirrer, and the solution was heated to $80{ }^{\circ} \mathrm{C}$ for 2 hours to hydrolyze the dianhydride to tetracarboxylic acid. After that, the solution was cooled down to room temperature, and equimolar amount of triamine TPAB (0.1291 g) was added, while the yellow salt suspensions were kept under stirring over night at room temperature.

\subsection{Hydrothermal synthesis of PI aerogels}

The above prepared monomer salt precursor solution was mixed with $3 \mathrm{ml}$ deionized water, the solution was further stirred for $1 \mathrm{~h}$ at room temperature. The freshly prepared salt dispersion was transferred to PTFE-lined steel autoclave. The autoclave was placed in an oven at $200{ }^{\circ} \mathrm{C}$ for $24 \mathrm{~h}$ to fulfill the imidization process. The resulting PI monoliths were washed with water, followed by oven drying at $100{ }^{\circ} \mathrm{C}$ to yield a pure PI aerogel.

\subsection{Hydrothermal synthesis of PI/GO nanohydrid aerogels}

Typically, the above prepared monomer salt precursor solution was mixed with $3 \mathrm{ml} \mathrm{GO}$ aqueous dispersion and stirring for $1 \mathrm{~h}$ at room temperature. The freshly prepared mixed solution was transferred to PTFE-lined steel autoclave, then the autoclave was placed in an oven at $200{ }^{\circ} \mathrm{C}$ for $24 \mathrm{~h}$ to fulfill the imidization process and resulted in PI/GO nanohybrid aerogels.

\subsection{Characterizations}

FT-IR spectra were measured with a Nicolet 560 spectrometer. Scanning electron microscopy (SEM) was conducted with an Inspect F SEM at an accelerating voltage of $20 \mathrm{kV}$. The nitrogen absorption and desorption isotherms were measured at $77 \mathrm{~K}$ using a Tristar system. The sample was degassed at $150{ }^{\circ} \mathrm{C}$ for 10 hours before the measurement. Surface area was calculated from the adsorption data using Brunauer-Emmett-Teller (BET) method. The thermal property was measured using a thermo gravimetric analysis (TGA) instruments (SDT Q600) over the temperature range of 30 to $800{ }^{\circ} \mathrm{C}$ with a heating rate of $10{ }^{\circ} \mathrm{C} \mathrm{min}^{-1}$. The compression test was performed on an Instron Corporation 5507 universal testing machine, stress-strain curves were measured at a strain rate of $10 \mathrm{~mm} \mathrm{~min}{ }^{-1}$.

\section{Results and discussion}

Traditionally, PIs were synthesized through a two-step method by dissolving dianhydride and diamine in NMP, DMAc or $\mathrm{DMF},{ }^{24}$ all of these solvent are seriously environmental pollution. We choose a green one-pot hydrothermal polymerization process using nylon-salt-type monomers as precursor. The nylon-salt-type monomer is extremely reactive and thus is a reasonable precursor for the preparation of polyimide. ${ }^{25,26}$ The process for the green hydrothermal preparation of PI aerogels is shown in Scheme 1, where the dianhydride was firstly hydrolyzed to corresponding tetracarboxylic acid, which was then reacts with triamine TAPB to form the nylon-salt precursor. Due to the poor solubility of TAPB in water, the solution was stirred overnight at room temperature to fulfill the salt formation.

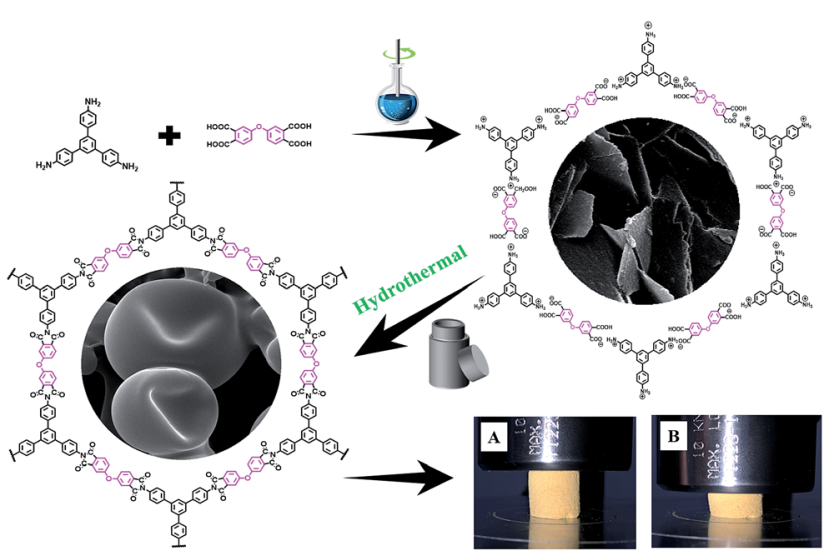

Scheme 1 Schematic presentation of soft/hard template directed dissolution-polymerization-reprecipitation process for the preparation of robust compressible PI aerogels using water as the only solvent. 

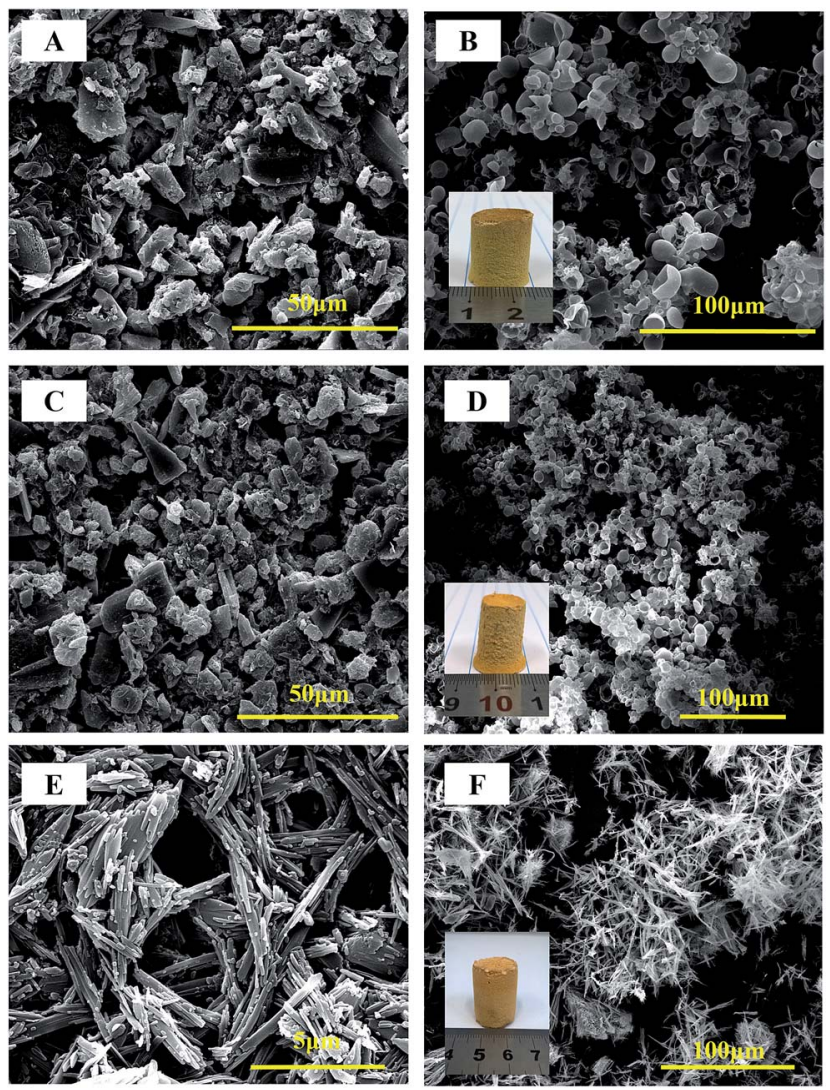

Fig. 1 SEM images of monomer salts (A, C, E) and SEM images and digital photos of PI aerogels ( $B, D, F)$ using different dianhydride as $\operatorname{ODPA}(A, B), \operatorname{BPDA}(C, D)$ and PMDA $(E, F)$.

Various dianhydrides such as ODPA, BPDA and PMDA was reacted with TAPB respectively, all the monomer salts showed crystalline appearance, as can be seen in SEM images in Fig. 1. $\mathrm{ODPA}^{2+}-\mathrm{TAPB}^{3-}$ and $\mathrm{BPDA}^{2+}-\mathrm{TAPB}^{3-}$ are polyhedrons, while $\mathrm{PMDA}^{2+}-\mathrm{TAPB}^{3-}$ are nanorods.

The monomer salt aqueous solution was transferred to a PTFE autoclave and heated to $200{ }^{\circ} \mathrm{C}$ for $24 \mathrm{~h}$ to finish the imidization process to form polyimide. Such a hydrothermal process has been intensively researched and generally PI particles or powders were obtained. However, when we chose a triamine to form network PI instead of linear PI, the results were totally changed, to our surprise, instead powder precipitates, after hydrothermal process, monolith compressible PI aerogels were obtained, the PI aerogels are mechanical robust which can be directly dried in oven without significant contraction (Fig. 1). To the best of our knowledge, this is the first example of highly cross-linked, fully aromatic polyimide aerogel showing a monolithic character and high compressive strength with reversibility, not to say we using water as the only solvent in the whole process.

A closed investigation using SEM revealed the morphologies of the hydrothermal synthesized PI aerogel are totally different. For PMDA-TAPB aerogel, loosely interconnected microfibers were observed, however, for ODPA-TAPB and BPDA-TAPB aerogel, interconnected hollow spheres were observed, some of the spheres were broken, and hollow interior can be clearly identified. Their morphologies have a direct influence on their macroscopic mechanical properties, among the three samples, ODPA-TAPB polyimide aerogel composed with uniform microhollow spheres possess the best compressibility and reversibility.

Most of the polymer aerogels reported so far are composed with interconnected micro/nano spheres or fibers, elucidate the origin of hollow spheres in PI aerogel is of great interest. We prefer a "spontaneous bubble-template" mechanism where the bubbles formed in situ during stirring were employed as a template for the growth of hollow spheres, this assumption can find support from the fact that if the nylon-salt-type monomers aqueous solution was degassed by vacuum for $1 \mathrm{~h}$, after hydrothermal imidization, solid sphere were obtained. While for the un-degassed sample, hollow spheres can be clearly observed from the early step of hydrothermal process (after 2 h) (Fig. 2).

The successful preparation of nylon-salt-type monomers and their corresponding PI aerogels was confirmed by FT-IR. The FT-IR spectra of all the nylon-salt-type monomers showed two broad arylammonium vibration modes around $2800 \mathrm{~cm}^{-1}$ and $2600 \mathrm{~cm}^{-1}$ as well as the $\mathrm{N}-\mathrm{H}$ vibration around $3400 \mathrm{~cm}^{-1}$ and $1470 \mathrm{~cm}^{-1}$. Moreover, the bands corresponding to carboxylic acids can be clearly identified around $1700 \mathrm{~cm}^{-1}$, (Fig. 3a). After hydrothermal process, all of the above mentioned peaks were almost diminished, the three PI aerogels showed similar patterns and characterized bands according to the cyclic imide vibrations can be clearly identified around $1784 \mathrm{~cm}^{-1}(\mathrm{C}=\mathrm{O}$, imide, asymmetric), $1722 \mathrm{~cm}^{-1}(\mathrm{C}=\mathrm{O}$, imide, symmetric), and $1370 \mathrm{~cm}^{-1}$ (C-N, imide symmetric).

Graphene oxide (GO) is hydrophilic and can be readily dispersed in water. A combination of GO with the hydrothermal
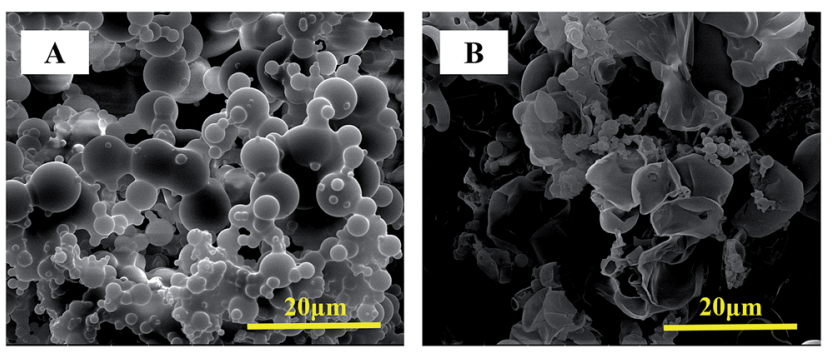

Fig. 2 SEM images of ODPA-TAPB aerogel: (A) the monomer salt was degassed for $1 \mathrm{~h}$ before hydrothermal treated for $24 \mathrm{~h}$. (B) Hydrothermal treated for only $2 \mathrm{~h}$ without degas.
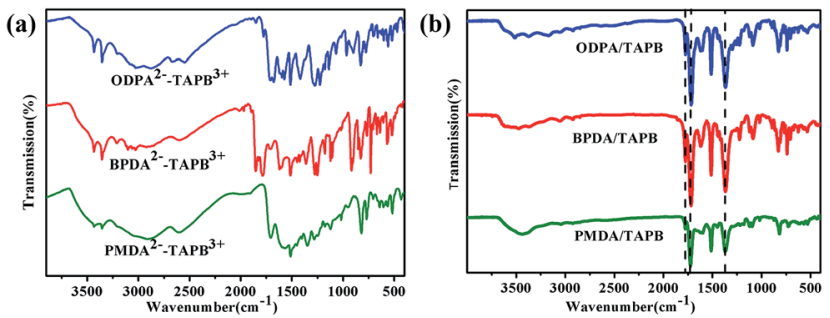

Fig. 3 FT-IR spectra of (A) nylon-salt-type monomers and (B) PI aerogel prepared from ODPA, BPDA and PMDA respectively. 
synthesis of PI might may pave a green approach to high performance nanohybrid materials. Thus various amount of aqueous GO solution was mixed with nylon-salt-type monomer solution, which was then hydrothermal treated at $200{ }^{\circ} \mathrm{C}$ to finish the imidization process. After hydrothermal process, monolith gray PI/GO nanocomposite aerogels with good mechanical properties were also formed, thus prove the generality of this hydrothermal process for the preparation of monolithic PI materials (Fig. 4).

Surprisingly, the microstructure of the nanohybrid aerogels is quite different from those of pure aerogels. When small amount of GO was added, the aerogel are still interconnected hollow spheres, however, small amount of nanofibers can be observed (Fig. 4A). With the increasing of GO, SEM revealed the aerogel was composed with very long and uniform PI nanofibers with diameter $50 \mathrm{~nm}$ growth on the surface of GO, thus formed an interesting 1D-2D nanohybrids (Fig. 4C). We follow the growth of nanofibers, after $2 \mathrm{~h}$, some small PI crystallites can be observed on the surface of GO, which will further growth to long and uniform nanofibers. The results clearly indicate that compared with gas bubbles, the GO possess a stronger interaction with monomer salts and thus can direct the polymerization induced assembly and formed unprecedented morphology transformation from hollow sphere to nanofibers.

In order to rationalize the green hydrothermal formation of rarely observed compressible PI aerogels with interconnected
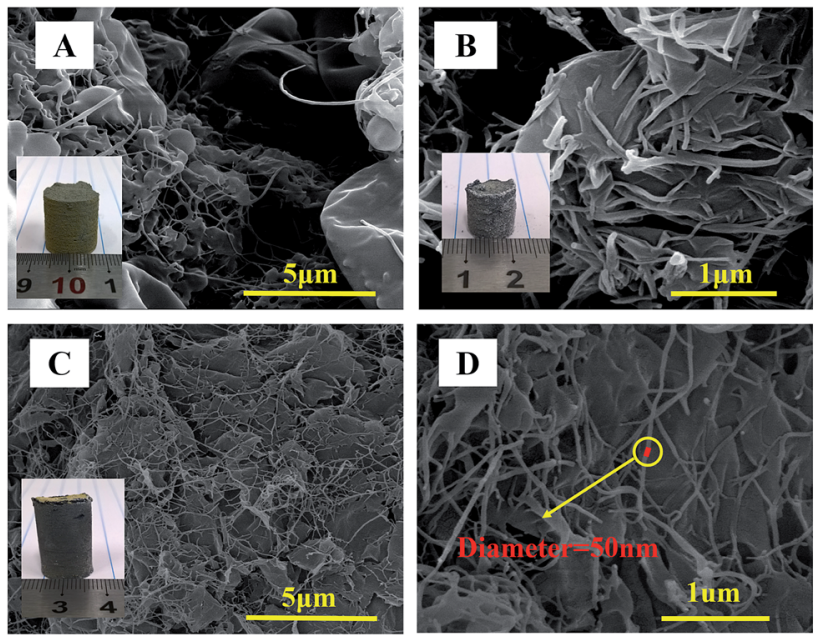

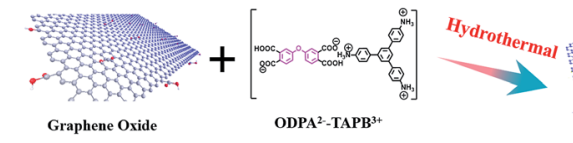



PI long nanofiber

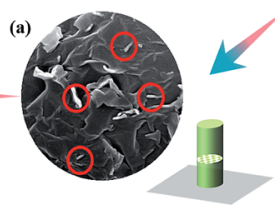

PI short nanofiber

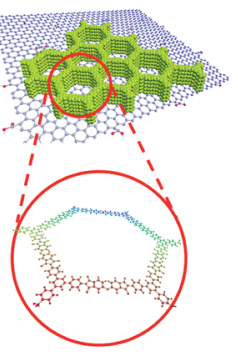

Fig. 4 SEM images of Pl/GO aerogels with $3 \mathrm{ml} G O$ aqueous solution: (A) 2, (B) 4 and (C) $8 \mathrm{mg} \mathrm{ml}^{-1}$, (D) local image of (C). Scheme for the $\mathrm{GO}$-directed growth of $\mathrm{PI}$ nanofibers on $\mathrm{GO}$.



Scheme 2 (above) Gas bubble directed formation of PI aerogel composed of interconnected hollow spheres, and (below) GO directed formation of 1D-2D PI nanofibers/GO nanohybrid aerogels.

hollow spheres or 1D-2D nanohybrid, a plausible formation mechanism based on soft/hard templates directed dissolutionpolymerization-reprecipitation process (DPRP) was proposed and can be illustrated as follow (Scheme 2): when the monomer salts solution was mechanically stirred overnight, many gas bubbles were formed in situ. In the followed hydrothermal process, small amount of the monomer salt will dissolved in the water, followed by hydrothermal polymerization to afford PI oligomers in the solution. When the degree of polymerization reached a critical value, they will precipitate from the solution and self-assembly on the air/water interface of gas bubbles. Repeated and rapid dissolution the monomer salt followed by polymerization and self-assembly precipitation lead ultimately to hollow microspheres, which are tightly interconnected to form the monolithic aerogels (Scheme 2A). With the existence of GO, when the degree of polymerization reached a critical value, the PI oligomers will precipitate from the solution and selfassembly on the GO surface instead of gas bubble interface due to their preferred interaction. The nucleation growth likes to take along the $1 \mathrm{D}$ direction, and gradually leading to the formation of pure uniform PI nanofibers growth on GO sheets (Scheme 2B).

The green synthesized PI aerogel were very robust and can be dried directly and thus avoid using laborious freezing drying or supercritical drying. In the completely dried state, the PI aerogel can quickly returned to its initial shape. Fig. 5 shows the compressive stress-strain curves of PI aerogel and PI/GO aerogel for the first and tenth cycles and the maximum stress changes during 10 cycles of loading-unloading tests. The hysteresis loops remained almost consistent, and the decrease in the maximum stress was negligible even after 10 cycles of loading-unloading. The stress-strain cures also revealed that small amount of GO greatly enhanced their compressive strength to one order $(14.5 \mathrm{kPa}$ to $220.6 \mathrm{kPa})$ without sacrificial their compressibility.

The prepared aerogels are amorphous, PXRD patterns revealed amorphous holo, this is reasonable because of their 

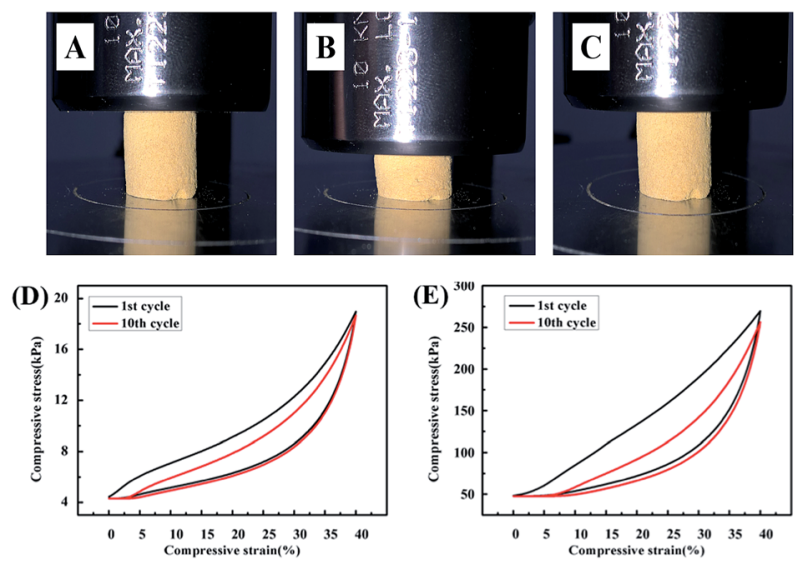

(F)
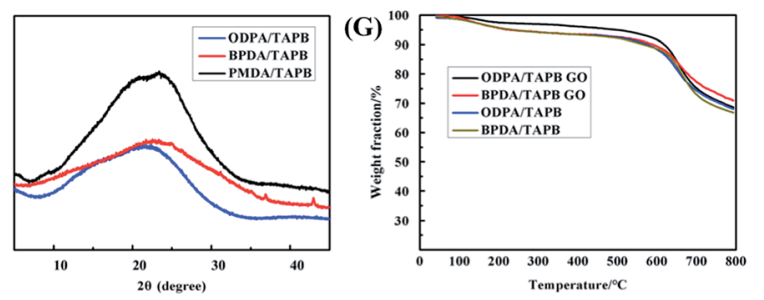

Fig. 5 Compressible properties of ODPA-TAPB PI aerogel (A-C), and stress-strain curves of (D) ODPA based PI aerogel and (E) PI/GO nanohybrid aerogel for the first (black) and tenth (red) test cycles. And (F) PXRD patterns and (G) TGA curves of the PI aerogels and PI/GO nanohybrid aerogels.

highly cross-linked framework structure. Due to the fully aromatic and rigid molecular backbone, the PI aerogels show outstanding thermal stability and high char yields. Temperatures of $10 \mathrm{wt} \%$ loss in $\mathrm{N}_{2}$ atmosphere were all above $600{ }^{\circ} \mathrm{C}$, with char yields at $800{ }^{\circ} \mathrm{C}$ over $65 \%$. Much effort has been devoted to explore new carbon aerogel materials with various morphologies and uniform microporous structures due to their high porosity and high stability. ${ }^{27-29}$ Due to their high char yields, the PI aerogels were carbonized at $800{ }^{\circ} \mathrm{C}$ for $2 \mathrm{~h}$, SEM revealed the original morphologies of the PI aerogel and nanohybrid aerogel were well maintained (Fig. 6A). The PXRD diffraction peaks are broad in shape. The Raman spectra (Fig. 6D) shows two main peaks at around $1400 \mathrm{~cm}$ and $1600 \mathrm{~cm}$ with the ratio of $I_{\mathrm{G}} / I_{\mathrm{D}}=1$, which is attributed to the amorphous and graphitic carbon phase.

The $\mathrm{N}_{2}$ sorption measurements of carbon aerogels were carried out at $77 \mathrm{~K}$ (Fig. 7). For the ODPA/TAPB carbon aerogel, the isotherm sorption profiles were observed with the typical type I characteristics, which indicative of a micropore character. The BET surface area were calculated as $575 \mathrm{~m}^{2} \mathrm{~g}^{-1}$, the value is just moderate, however, the micropore area is as high as $563 \mathrm{~m}^{2}$ $\mathrm{g}^{-1}$, which indicating that carbon aerogel was consisted with dominantly microporous structures. More impressively, the pore size distributions calculated by NLDFT model was found to have a predominant distribution centred at $0.8 \mathrm{~nm}$ (Fig. 7A), to the best of our knowledge, most carbon aerogels carbonized from MOFs, COFs or CMPs have rather broad pore size distributions spread nearly from micropore to mesopore, ${ }^{30-34}$ the accurate control of pore properties of is hard to achieve due to
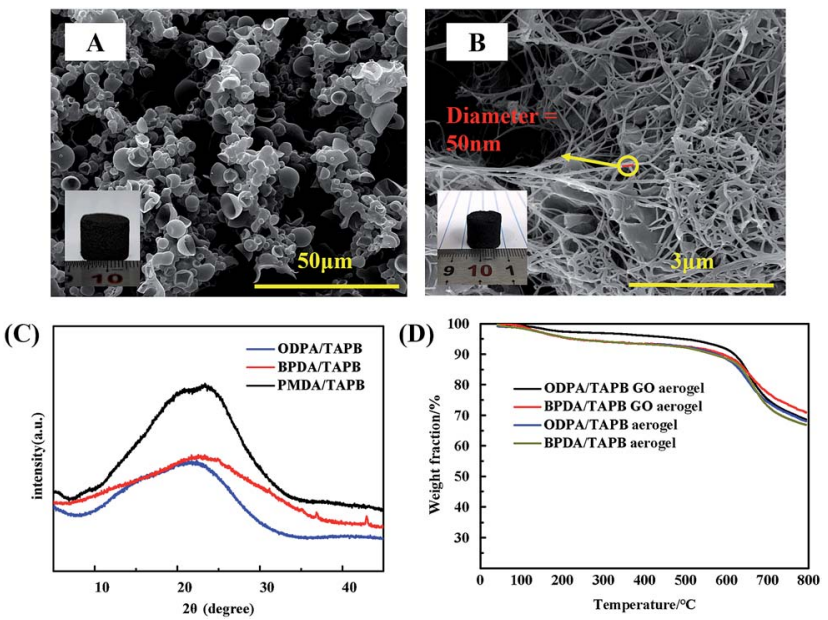

Fig. 6 SEM and digital photo of pure carbon aerogels (A) and PI/GO nanohybrid aerogels (B), the (C) PXRD patterns and (D) Raman spectra of the carbon aerogels, carbon aerogel (a), carbon aerogel using $3 \mathrm{ml}$ ( $2 \mathrm{mg} \mathrm{ml}^{-1}$ ) (b) and $3 \mathrm{ml}\left(8 \mathrm{mg} \mathrm{ml}^{-1}\right)$ (c) GO.

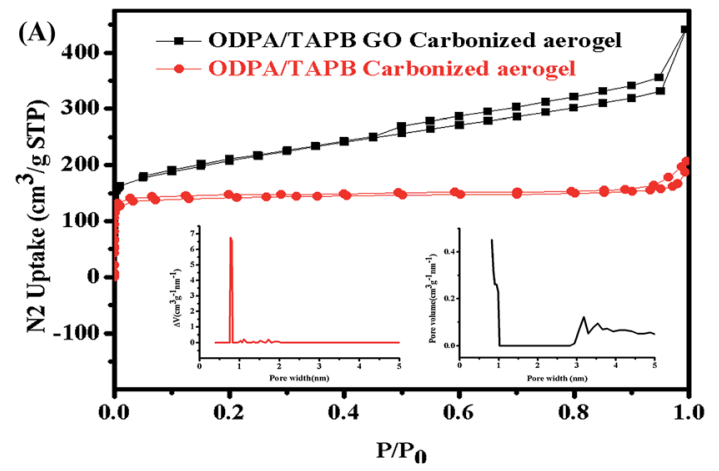

(B)
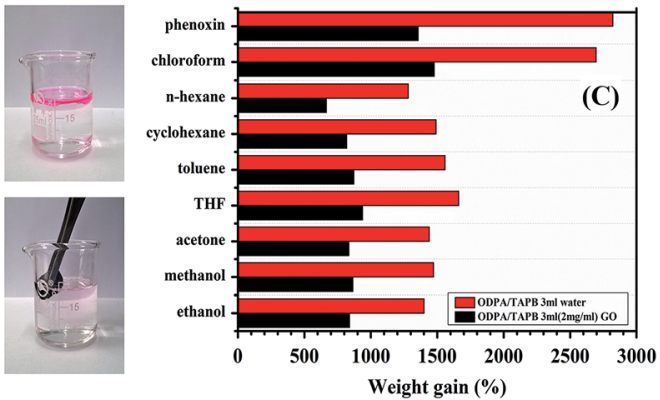

Fig. 7 (A) $\mathrm{N}_{2}$ adsorption-desorption isotherms of ODPA based pure and nanohybrid carbon aerogels. (B) Before and the removal of toluene from water using ODPA based carbon aerogel, and (C) absorption capacity of carbon aerogel for various liquids.

the large framework contraction involve in high temperature carbonization process. In contrast to these earlier reports, our results revealed that a near monodisperse micropore size could be formed in the monolithic carbon aerogel. A plausible reason may be due to the that the aerogel precursor was composed with PI which is the most rigid, toughest member of the heatresistance aromatic polymers with extremely high carbon yields. The introduce of GO leading to a nitrogen sorption 
isotherm type IV, with a BET special surface area of $741 \mathrm{~m}^{2} \mathrm{~g}^{-1}$, which indicating that a mixture of micropores and mesopores are present in the nanohybrid carbon aerogel.

The microporous properties of these monolithic PI aerogels made it a good candidate for the removal of pollutants from an aqueous solution. The gravimetric absorption capacity of PI aerogel for various liquids was plotted, as shown in Fig. 7. The absorption capacities were in the range of 600 to $2800 \mathrm{wt} \%$ depending on the density of the absorbate. More specific applications using the uniform microporosity, electrical conductivity, chemical stability and thermal stability was in progress.

\section{Conclusion}

In recent decade, the scientific community has great interest in developing material synthesis that minimizes the environmental impact. Here through a facile one-pot hydrothermal process, we reported the first green approach to the morphological controlled synthesis of fully aromatic polyimide aerogels and their graphene nanohybrid aerogel using water as the only solvent. Robust, compressible PI aerogels composed with tightly interconnected hollow micro spheres were synthesized using in situ formed gas bubble as soft templated, and PI aerogel composed with 1D-2D structures were synthesized through graphene oxide directed growth of PI nanowires. The mechanism behind is a soft/hard template directed dissolution-polymerization-reprecipitation process. Such a hydrothermal polymerization process kills two birds with only one stone: obtaining monolithic PI aerogels with excellent thermal and mechanical properties, and doing so without using any kind of organic solvent but hot water. After carbonization, monolith carbon aerogel with unique morphologies and monodisperse micropore size could be formed. Such a green approach for the morphology controlled synthesis of robust polyimide aerogel and their carbon aerogels should be of great value for their applications in environmental or energy related applications.

\section{Acknowledgements}

We are grateful for the financial support from the National Natural Science Foundation of China (No. 21174089, 21674068 and 51633004).

\section{References}

1 S. S. Kistler, Nature, 1931, 127, 741.

2 H. Hu, Z. B. Zhao, W. B. Wan, Y. Gogotsi and J. S. Qiu, Adv. Mater., 2013, 25, 2219-2223.

3 Z. L. Yu, G. C. Li, N. Fechler, N. Yang, Z. Y. Ma, X. Wang, M. Antonietti and S. H. Yu, Angew. Chem., Int. Ed., 2016, 55, 14623-14627.

4 J. P. Randall, M. A. B. Meador and S. C. Jana, ACS Appl. Mater. Interfaces, 2011, 3, 613-626.

5 R. W. Pekala, J. Mater. Sci., 1989, 24, 3221-3227.

6 G. Biesmans, A. Mertens, L. Duffours, T. Woignier and J. Phalippou, J. Non-Cryst. Solids, 1998, 225, 64-68.
7 W. Zhang, Y. A. Zhang, C. H. Lu and Y. L. Deng, J. Mater. Chem., 2012, 22, 11642.

8 J. Hassoun, F. Bonaccorso, M. Agostini, M. Angelucci, M. G. Betti, R. Cingolani, M. Gemmi, C. Mariani, S. Panero, V. Pellegrini and B. Scrosati, Nano Lett., 2014, 14, 4901-4906.

9 D. Yang, L. Y. Zhou, L. C. Chen, B. Zhao, J. Zhang and C. Li, Chem. Commun., 2012, 48, 8078-8080.

10 N. Vaenas, D. Konios, T. Stergiopoulosc and E. Kymakis, RSC Adv., 2015, 5, 107771-107776.

11 C. Chung, Y. K. Kim, D. Shin, S. R. Ryoo, B. H. Hong and D. H. Min, Acc. Chem. Res., 2013, 46, 2211-2224.

12 V. Tozzini and V. Pellegrini, Phys. Chem. Chem. Phys., 2013, 15, 80-89.

13 A. Javadi, Q. F. Zheng, F. Payen, A. Javadi, Y. Altin, Z. Y. Cai, R. Sabo and S. Q. Gong, ACS Appl. Mater. Interfaces, 2013, 5, 5969-5975.

14 L. Z. Zuo, W. Fan, Y. F. Zhang, L. S. Zhang, W. Gao, Y. P. Huang and T. X. Liu, Compos. Sci. Technol., 2017, 139, 57-63.

15 M. A. Aegerter, N. Leventis and M. M. Koebel, Aerogels Handbook, Springer, New York, USA, 2011.

16 M. A. B. Meador, E. J. Malow, R. Silva, S. Wright, D. Quade, S. L. Vivod, H. Q. Guo, J. Guo and M. Cakmak, ACS Appl. Mater. Interfaces, 2012, 4, 536-544.

17 H. Q. Guo, M. A. B. Meador, L. McCorkle, D. J. Quade, J. Guo, B. Hamilton, M. Cakmak and G. Sprowl, ACS Appl. Mater. Interfaces, 2011, 3, 546-552.

18 H. Q. Guo, M. A. B. Meador, L. McCorkle, D. J. Quade, J. Guo, B. Hamilton and M. Cakmak, ACS Appl. Mater. Interfaces, 2012, 4, 5422-5429.

19 B. Baumgartner, M. J. Bojdysb and M. M. Unterlass, Polym. Chem., 2014, 5, 3771-3776.

20 B. Baumgartner, M. Puchberger and M. M. Unterlass, Polym. Chem., 2015, 6, 5773-5781.

21 M. M. Unterlass, D. Kopetzki, M. Antonietti and J. Weber, Polym. Chem., 2011, 2, 1744-1753.

22 K. Kriechbaum, D. A. Cerroń-Infantes, B. Stöger and M. M. Unterlass, Macromolecules, 2015, 48, 8773-8780.

23 J. Lee and J. Y. Chang, Chem. Commun., 2016, 52, 1041910422.

24 D. Wilson, H. D. Stenzenberger and P. Hergenrother, Polyimides, Blackie \& Son Ltd., Glasgow and London, 1990.

25 T. Inoue, Y. Kumagai, M. Kakimoto, Y. Imai and J. Watanabe, Macromolecules, 1997, 30, 1921-1928.

26 Y. Imai, Adv. Polym. Sci., 1999, 140, 1-22.

27 S. B. Yang, R. E. Bachman, X. L. Feng and K. Mullen, Acc. Chem. Res., 2013, 46, 116-128.

28 L. F. Chen, X. D. Zhang, H. W. Liang, M. G. Kong, Q. F. Guan, P. Chen, Z. Y. Wu and S. H. Yu, ACS Nano, 2012, 6, 70927102.

29 L. Hao, B. Luo, X. L. Li, M. H. Jin, Y. Fang, Z. H. Tang, Y. Y. Jia, M. H. Liang, A. Thomas, J. H. Yang and L. J. Zhi, Energy Environ. Sci., 2012, 5, 9747-9751.

30 J. S. M. Lee, M. E. Briggs, T. Hasell and A. I. Cooper, $A d v$. Mater., 2016, 28, 9804-9810. 
31 K. Yuan, T. Hu, Y. Z. Xu, R. Graf, G. Brunklaus, M. Forster, Y. W. Chen and U. Scherf, ChemElectroChem, 2016, 3, 822828.

32 Y. H. Shih, C. P. Fu, W. L. Liu, C. H. Lin, H. Y. Huang and S. Ma, Small, 2016, 12(15), 2057-2066.
33 K. Xi, S. Cao, X. Y. Peng, C. Ducati, R. V. Kumar and A. K. Cheetham, Chem. Commun., 2013, 49, 2192-2194.

34 A. J. Amali, J. K. Sun and Q. Xu, Chem. Commun., 2014, 50, 1519-1522. 